\title{
Design of Geological Hazard Monitoring System
}

\author{
Guoshao Chen, Zhongsheng Wang
}

School of Computer Science and Engineering, Xi'an Technological University,

Xi'an 710021, P.R.China

1825247141@qq.com,59483672@qq.com

\section{Keyword: Geological hazard; GPRS}

\begin{abstract}
Geological hazards such as landslides, debris flow and so on are not stable in the Qinling Mountains range. It is necessary to forecast the geological disaster, how to collect the data and transfer the data to the data center in real time. This paper introduces the design of integrated geological hazard monitor, including hardware structure design, software design and database design. After the initial operation, the system is stability and accuracy to meet the demand. The system uses high-power solar power, to ensure that power is not subject to power failure. Data transmission using GPRS, making the difficulty of the construction greatly reduced.
\end{abstract}

\section{Introduction}

In 2015, landslide disaster in Shanyang County, resulting in 65 deaths or missing, Qinba mountain geological disaster mechanism is complex, at any time there may be the occurrence of disaster.

In recent years, Shaanxi land and resources departments to strengthen the monitoring and early warning, and to strengthen the knowledge of geological disasters, publicity, public awareness and self-help in disaster prevention and reduction capacity has improved significantly. Through the organization of group measurement group defense way to successfully predict or avoid geological disasters to avoid losses increase year by year. However, due to the lack of monitoring equipment, can not be real-time changes in the scene, there is no data on-site forecast basis. In this paper, a practical geological hazard monitoring instrument is designed to monitor geological deformation in real time so as to predict disaster in advance and prepare for disaster prevention.

\section{System Overall Design}

System consists of a lot of data acquisition terminal and data server. Data acquisition terminal to collect data and transfer to internet. The data server receives the data from the Internet and saves it,
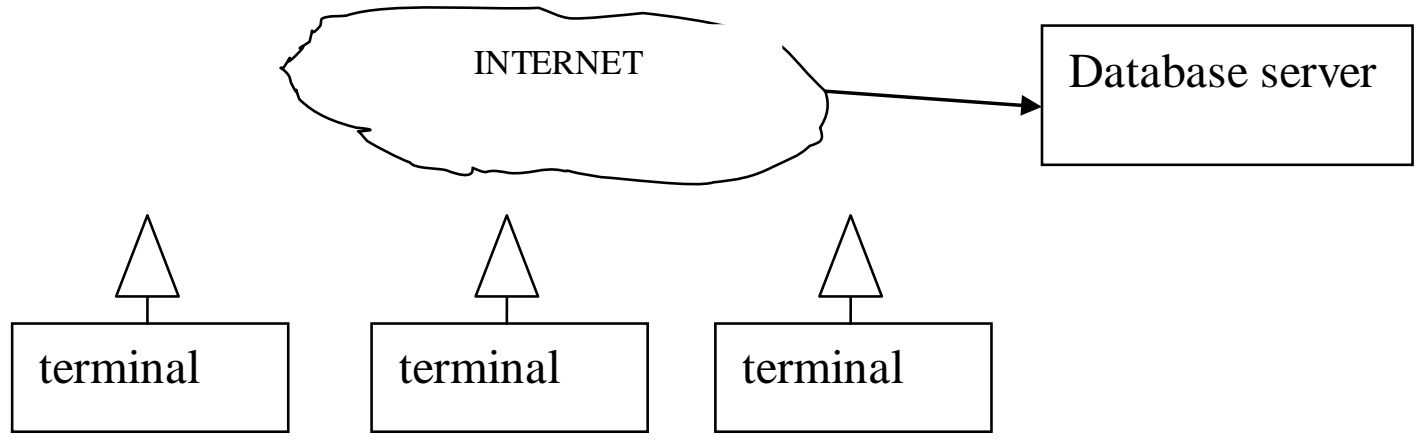

Figure 1. The overall structure of the system

Calculates and judges whether the alarm is work. The overall structure shown in Fig. 1.

\section{Hardware Design}

The potential landslide body usually moves very little, once the condition is ripe, the rapid movement causes the disaster. Disasters often occur during stormy periods, and heavy rain often 
leads to disruption of electricity supply. In order to ensure uninterrupted power supply, the design of solar panels and lead-acid batteries combined power supply. According to the characteristics of geological disasters in Qinba mountainous area, the system design meets the requirements of high precision, real-time and low power consumption. System power supply using solar cells combined with large-capacity battery-powered to ensure uninterrupted work of monitoring equipment. Displacement sensor accuracy of $0.1 \mathrm{~mm}$, power consumption as low as $1 \mathrm{mw}$. Monitoring area not far from the residential area, the basic coverage by the mobile communication network, the equipment used GPRS communications, construction costs are low, the construction convenient. Equipment comes with LCD screen, real-time display crack width. The hardware structure shown in Fig. 2.

System use stc12le5a60s2 as the main processor, which is a low-power low-voltage processor, the operating speed is the same frequency conventional microcontroller 8 times to support idle time sleep, more energy savings. The processor has $60 \mathrm{k}$ bytes of flash program memory and $2 \mathrm{k}$ bytes of EEPROM. LCD screen with ultra-low power monochrome LCD screen LCD5110, idle time off the backlight to save power. The sensor converts the crack width into a voltage signal, and the voltage signal is converted to a 16-bit digital signal by the analog-to-digital converter ADS1110. The accuracy of the voltage signal can meet the requirements of the system. Solar panels maximum power of 20 watts, quickly charge the battery. The sensor adopts the high accuracy displacement sensor, the structure is simple and reliable, and the precision is not influenced by the outside temperature. The precision is $0.1 \mathrm{~mm}$, which can meet the requirements of the on-site crack monitoring.

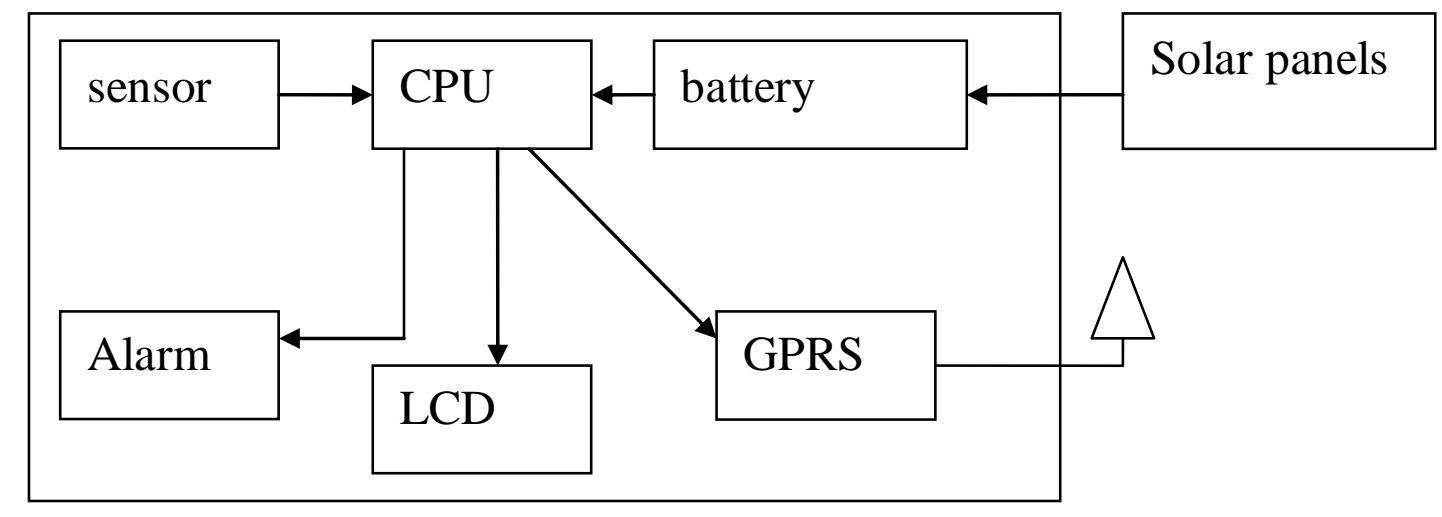

Figure 2. Hardware structure

The working process of the system is as follows: When the sensor displacement exceeds the threshold, the CPU computes the result to the LCD screen. At the same time, the data is sent to the host computer through GPRS communication module. The default is sent to three positions, namely, county, server. If the data exceeds the alarm threshold, start the alarm.

\section{Software Design}

The Main Program Design. Main program includes system initialization, time update, data acquisition, data transmission and preservation, to determine the alarm and other parts. Its structure as shown in Fig. 3. 


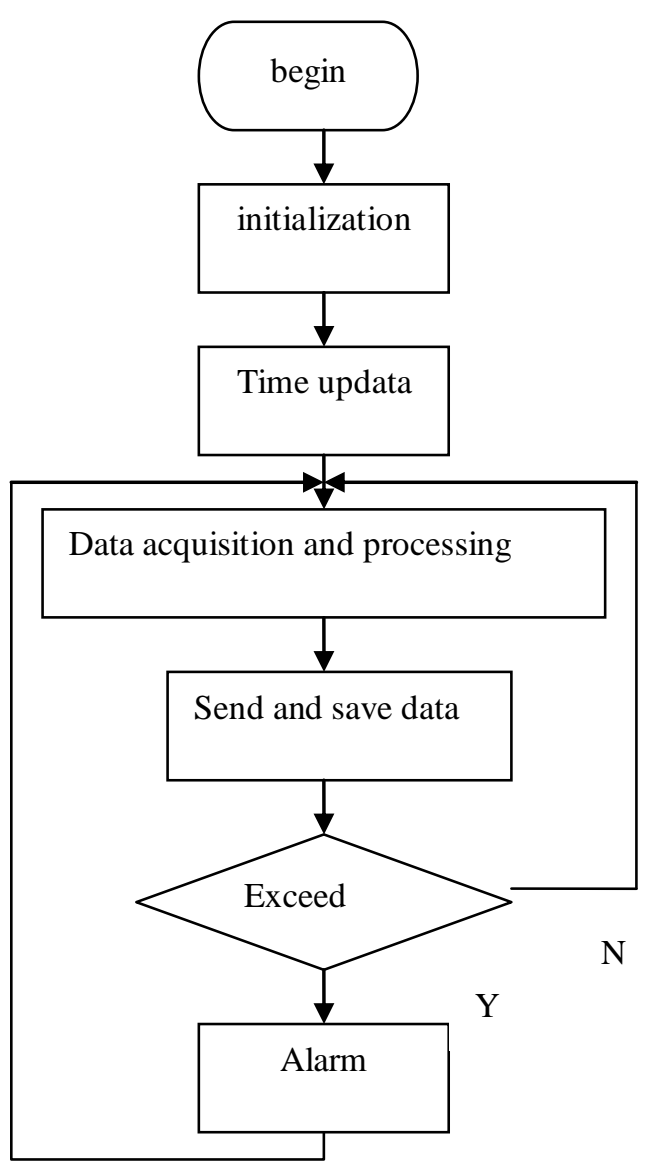

Figure 3. Software overall structure

System initialization after power-on settings, including timer cycle settings, communication speed, communication mode settings, GPRS work settings. When the system starts, the clock chip time is not accurate; you need to get accurate from the network time. In order for all acquisition units to be synchronized, the acquisition unit clock is based on the server time. After all the settings are complete, the system collects the data and obtains the sensor data through the A / D converter. The data is sent to the server via the GPRS network and saved to the memory. After the data processing is completed, the system reads the threshold and compares it. When the crack width exceeds the threshold, the alarm is activated.

The Important Subroutine Design. Data is the basis for decision-making and alarm, therefore, the accuracy and real-time data is particularly important. The precision of the sensor is $0.1 \mathrm{~mm}$, the precision of the analog / digital converter is 16 , and the precision can meet the requirement. In order to prevent the impact of field interference on the data, the data to digital filter processing. The system collects 100 sets of data for each collection cycle. The maximum 10 values and the minimum 10 values are removed. The remaining 80 data are averaged. Through the filter can remove the interference, to ensure accurate data.

The Data Transmission Format. As the data transmission using GPRS, the data to the server has a certain delay, in order to get the accurate time of data, the data including acquisition time. Each data include valid data, time, alarm level and other basic points. The time is the collection of data of the time. Address is the address of the acquisition unit, four bytes. The fracture length data is five bytes, unit is millimeters. Alarm level refers to the current alarm level, 0 is not alarm, the other three levels are for alarm.

\section{Database Management System Design}

The data collected by the data acquisition terminal is transmitted to the database server through GPRS, in order to make the data reliable, CRC check is used. Here the communication program is 
relatively simple, call the system control and modify the parameters. Database system detect network data in real time, $\mathrm{CRC}$ check if the return is not correct, the data is discarded, otherwise the data extracted and saved to the database, at the same time compared with the alarm threshold, if exceeds a threshold start alarm. The CRC check function is as follows:

int calcByte (int CRC, char b) / / this function is used for bit operation, provide functions for the following calculation

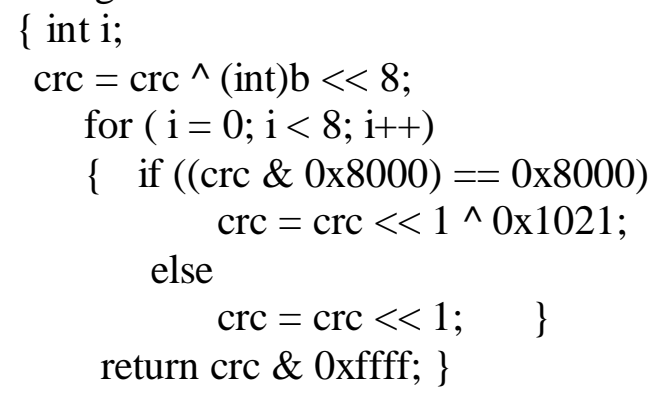

int CRC16 (char *pBuffer, int length) // 16 CRC code calculation function, the first argument is by the parity array, the second parameter is the length of the array, the return value is 16 CRC check code

$\{$ int $\mathrm{wCRC} 16=0$

int $\mathrm{i}$;

for $(\mathrm{i}=0 ; \mathrm{i}<$ length; $\mathrm{i}++)$

\{ wCRC16 = calcByte $($ wCRC16, pBuffer[i] $) ;\}$

return wCRC16;

\}

\section{Conclusion}

The hazard monitor has the following advantages. The structure is simple and reliable. The system uses the resistance type sensor, is not affected by the temperature, can guarantee the data has the very good stability. Data accuracy is high. Data accuracy of $0.1 \mathrm{~mm}$, fully meet the on-site measurement accuracy requirements. Construction convenient. Data transmission using GPRS, power supply using solar energy, making the acquisition unit and the outside world there is no cable connection.

After more than a year of experiments, the system data is stable, high precision, to meet the early warning requirements.

\section{References}

[1] YAN Xueping, et al. The development and application of remote data acquisition system based on the GPRS. Development and Application of Single Chip Microcomputer.

[2] YUAN Shenggui, et al. Application of the GPRS Technology in Mountainous Landslide of the Disaster Area. Application of Automation Technology and Application. 